\title{
The Use of Fractional B-Splines Wavelets in Multiterms Fractional Ordinary Differential Equations
}

\author{
X. Huang and X. Lu \\ School of Mathematical and Computer Sciences, Fuzhou University, Fuzhou 350002, China \\ Correspondence should be addressed to X. Huang,hxdjwan@163.com
}

Received 31 July 2009; Revised 2 November 2009; Accepted 4 November 2009

Academic Editor: Fawang Liu

Copyright (c) 2010 X. Huang and X. Lu. This is an open access article distributed under the Creative Commons Attribution License, which permits unrestricted use, distribution, and reproduction in any medium, provided the original work is properly cited.

We discuss the existence and uniqueness of the solutions of the nonhomogeneous linear differential equations of arbitrary positive real order by using the fractional B-Splines wavelets and the MittagLeffler function. The differential operators are taken in the Riemann-Liouville sense and the initial values are zeros. The scheme of solving the fractional differential equations and the explicit expression of the solution is given in this paper. At last, we show the asymptotic solution of the differential equations of fractional order and corresponding truncated error in theory.

\section{Introduction}

Recently, there have been several schemes devoted to the solution of fractional differential equations. These schemes can be broadly classified into two classes, numerical and analytical ([1]). As we know, with the help of some special functions, such as Mittag-Leffler function and Green function, Miller and Ross have obtained the explicit representations of solutions of some classes of homogeneous linear fractional differential equations (FDEs) in [2]; through the use of the technique of Laplace and Fourier Transform, the analytical solutions have been given by Podlubny in [3]. The numerical scheme we have encountered can be divided into two groups. In the first group, the solution is approximated over the entire domain using approximating functions such as polynomials and orthogonal functions. In the second group, the entire domain is divided into several small domains like in a finite element technique, and the solution is obtained for variables at the node points ([1]). As Edwards et al. declaimed in [4], several forms of fractional differential equations have been proposed in standard models, and there has been significant interest in developing numerical schemes for their solution. Thus, several papers have been presented in dealing with approximate numerical techniques 
for FDEs. Among these, the papers of Diethelm, Edwards, Ford, Freed, and Simpson are noteworthy (see, e.g., $[1,4-6]$ ). One of these schemes which should be mentioned is the use of a Predictor-Corrector (more precisely, PECE) method in [6]. In order to obtain higher precision, they have replaced the PECE method by a $\mathrm{P}(\mathrm{EC})^{m} E$ method with $m \geq 2$ in [7]. In particular, the PECE method is an important numerical scheme which has been applied in many fields; for example, Yang and Liu have applied the PECE method for simulating fraction order dynamical control system in [8]. In addition, several numerical schemes have also been proposed by other authors (see $[9,10])$. In this paper, we present a new scheme which contains the features of both two groups by using the fractional B-splines wavelet. In paper [11], Unser and Blu have proved that the fractional B-splines generates a valid multiresolution analyses of $L^{2}$ for $\alpha>-1 / 2$, which means that the orthogonal fractional Bsplines could be obtained by applying the standard technique described in [12]. And they also obtained that the fractional B-splines deriving the asymptotic development of the $L^{2}$ have a fractional order of approximation.

In fact, as the theories of wavelets analyses improve day by day, the wavelet has become a powerful mathematical tool which widely used in signal processing, image compression and enhancement, pattern recognition, control systems, and other fields in the past two decades. But almost no papers or books have applied the theories of wavelets to solve the fractional differential equations. And our fundamental purpose of this paper is applying the fractional B-Splines wavelets to prove the existence and uniqueness of the solution of the nonhomogeneous linear fractional differential equations (also so-called linear multiterm fractional differential equations) with its initial conditions. Let us begin to discuss the solution of multiterms fractional ordinary differential equations with the following form:

$$
\left(b_{n} D^{\alpha_{n}}+b_{n-1} D^{\alpha_{n-1}}+\cdots+b_{1} D^{\alpha_{1}}\right) y(t)=f(t) .
$$

For convenience, we consider the initial values:

$$
\left[D^{\alpha_{k}-1} y(t)\right]_{t=0}=0,\left[D^{\alpha_{k}-2} y(t)\right]_{t=0}=0, \ldots,\left[D^{\alpha_{k}-r_{k}} y(t)\right]_{t=0}=0
$$

where $D^{\alpha}$ are taken in the Riemann-Liouville sense, $\alpha_{n}>\alpha_{n-1}>\cdots>\alpha_{1}, b_{n} \neq 0, \alpha_{n} \geq 1$, and $0 \leq r_{k}-1 \leq \alpha_{k}<r_{k}, r_{k} \in N, \alpha_{k} \in R^{+}, b_{k} \in R, k=1, \ldots, n$. The function $f(t)$ belongs to the space $L^{2}(\Omega)$; without loss of generality, in this paper, we consider the interval as $\Omega=$ $[0, T], T \in R^{+}$.

The plan of this paper is as follows. In Section 2, we recall the definitions of fractional derivative and integral and related properties which will be used in this paper, give the representation of Mittag-Leffler function and generalized Mittag-Leffler function, and then introduce the fractional B-splines and some related properties of wavelets. In Section 3, by applying the technique of the Laplace Transforms, and considering the proprieties of the generalized Mittag-Leffler function, we prove the lemma of the differential equations of arbitrary positive real order, which make sure the solution belongs to the space $L^{2}$; by virtue of the solution that can be expressed as the form of wavelet series and the basis function that is the orthogonal fractional B-splines wavelet which yields the Riesz basis for the space $L^{2}$, we can prove the uniqueness of the coefficients of the representation of the solution, which gain the uniqueness of the solution of the fractional differential equations and validate the representation of solution. Thus, we have finished the proof of the theorem. 
In Section 4, the asymptotic solution of the differential equations of fractional order $\alpha \in Q^{+}$ and corresponding truncated error will be discussed.

The present paper is essentially based on the works of the Unser and Blu in [11] and Podlubny in [3], to which more general classes of the fractional differential equations we shall refer in the following research. For more related review of fractional B-spline wavelet, see the papers of the Unser and Blu $([11,13,14])$, which have discussed some more important wavelet properties, such as Riesz bounds and two-scale relation.

\section{Preliminary and Definitions}

\subsection{Definitions}

We may recall the definition of the Left Riemann-Liouville differential operators of arbitrary order $\alpha>0$, which take the form

$$
{ }_{0} D_{t}^{\alpha} y(t):=\frac{1}{\Gamma(m-\alpha)} \frac{d^{m}}{d t^{m}} \int_{0}^{t} \frac{y(\tau)}{(t-\tau)^{\alpha-m+1}} d \tau
$$

where $m$ is the integer defined by $m-1 \leq \alpha<m$ (see [3]), and $\Gamma(\cdot)$ is gamma function. Similarly, the left Riemann-Liouville integral operators of order $\alpha>0$ is defined as

$$
{ }_{0} D_{t}^{-\alpha} y(t):=\frac{1}{\Gamma(\alpha)} \int_{0}^{t}(t-\tau)^{\alpha-1} y(\tau) d \tau
$$

And then we should give the following expression for the Laplace transform of the RiemannLiouville differential operators of the order $\alpha>0$, which is

$$
L\left\{{ }_{0} D_{t}^{\alpha} y(t) ; s\right\}=s^{\alpha} Y(s)-\sum_{k=0}^{m-1} s^{k}\left[{ }_{0} D_{t}^{\alpha-k-1} y(t)\right]_{t=0^{\prime}} \quad(m-1 \leq \alpha<m) .
$$

Let us now introduce the case of the Caputo differential operators of arbitrary order $\alpha>0$, which is defined as

$$
{ }_{0}^{C} D_{t}^{\alpha} f(t)=\frac{1}{\Gamma(n-\alpha)} \int_{0}^{t} \frac{f^{(n)}(\tau) d \tau}{(t-\tau)^{\alpha+1-n}}, \quad(n-1<\alpha \leq n) .
$$

And the formula of its Laplace transform can be expressed as

$$
L\left\{{ }_{0}^{C} D_{t}^{\alpha} f(t) ; s\right\}=s^{\alpha} F(s)-\sum_{k=0}^{n-1} s^{\alpha-k-1} f^{(k)}(0), \quad(n-1<\alpha \leq n) .
$$

\subsection{Mittag-Leffler Functions and Generalized}

The Mittag-Leffler functions and its generalized forms have played a special role in solving the fractional differential equations. In this section, we just give the definition of the following 
series of representation of the Mittag-Leffler function $E_{\alpha}(z)$ with $\alpha>0$, which validates in the whole complex plane:

$$
E_{\alpha}(z):=\sum_{n=0}^{+\infty} \frac{z^{n}}{\Gamma(\alpha n+1)}, \quad \alpha>0, z \in C
$$

And for the generalized Mittag-Leffler function, we use the following definition:

$$
E_{\alpha, \beta}(z):=\sum_{n=0}^{+\infty} \frac{z^{n}}{\Gamma(\alpha n+\beta)}, \quad \alpha>0, \beta>0, z \in C
$$

Let us now consider the Laplace transforms of the function $t^{\alpha k+\beta-1} E_{\alpha, \beta}\left( \pm a t^{\alpha}\right)$ (see [3]), which is defined by

$$
\int_{0}^{\infty} e^{-s t} t^{\alpha k+\beta-1} E_{\alpha, \beta}^{(k)}\left(a t^{\alpha}\right) d t=\frac{k ! s^{\alpha-\beta}}{\left(s^{\alpha}-a\right)^{k+1}}, \quad\left(\operatorname{Re}(s)>|a|^{1 / \alpha}\right) .
$$

\subsection{Fractional B-Splines Wavelet}

Splines have had a significant impact on the early development of the theory of the wavelet transform (see [13]). And Unser and Blu have first mentioned the fractional B-Splines in [13], who extended Schoenberg's family of polynomial splines to all fractional degrees $\alpha>-1$ and defined the fractional causal B-splines by taking the $(\alpha+1)$ th fractional difference of the onesided power function:

$$
\beta_{+}^{\alpha}(x):=\frac{1}{\Gamma(\alpha+1)} \triangle_{+}^{\alpha+1} x_{+}^{\alpha}=\frac{1}{\Gamma(\alpha+1)} \sum_{k \geq 0}(-1)^{k}\left(\begin{array}{c}
\alpha+1 \\
k
\end{array}\right)(x-k)_{+}^{\alpha},
$$

where the one-side power function $x_{+}^{\alpha}$ is defined as follows:

$$
x_{+}^{\alpha}= \begin{cases}x^{\alpha}, & x \geq 0 \\ 0, & \text { otherwise }\end{cases}
$$

Then we introduce the fractional B-splines autocorrelation sequence as follows:

$$
\beta_{*}^{2 \alpha+1}(k):=\left\langle\beta^{\alpha}(x), \beta^{\alpha}(x-k)\right\rangle .
$$

From [13], we know the explicit form of the fractional B-splines wavelet:

$$
\psi_{+}^{\alpha}\left(\frac{x}{2}\right)=\sum_{k \in Z} \frac{(-1)^{k}}{2^{\alpha}} \sum_{l \in Z}\left(\begin{array}{c}
\alpha+1 \\
l
\end{array}\right) \beta_{*}^{2 \alpha+1}(l+k-1) \beta_{+}^{\alpha}(x-k),
$$


which yields a Riesz basis for $L^{2}(\Omega)$. Thus, using the standard orthogonalize technique described in [12], we have

$$
\hat{\varphi}_{+}^{\alpha}(\omega)=\frac{\sqrt{2} \hat{\psi}_{+}^{\alpha}(w)}{\left(\sum_{k=0}^{\infty}\left|\hat{\psi}_{+}^{\alpha}(\omega+2 k \pi)\right|^{2}\right)^{1 / 2}}
$$

where $\hat{\psi}_{+}^{\alpha}(w)$ is the Fourier Transform of the function of $\psi_{+}^{\alpha}(x)$. Thus we obtain the orthogonal fractional B-splines $\varphi_{+}^{\alpha}(x)$ which also yields a Riesz basis for $L^{2}(\Omega)$. According to the theories of wavelet analyses, the function $y(t) \in L^{2}(\Omega)$ can be expressed as

$$
y(t)=\sum_{k=-\infty}^{+\infty} c(k) \varphi_{+}^{\alpha}(t-k),
$$

where the coefficients $c(k)$ are constants.

\section{The Existence and Uniqueness of the Solution of Multiterms Fractional Ordinary Differential Equations}

In this section, we will prove the existence and uniqueness properties of the solutions of the nonhomogeneous linear differential equations of arbitrary real order $\alpha>0$.

\subsection{An Important Lemma}

Under the hypothesis of the existence for the solutions of (1.1), we have the following lemma.

Lemma 3.1. Let $\alpha_{n}>\alpha_{n-1}>\cdots>\alpha_{1}$, and $\alpha_{n} \geq 1$, and $f(t) \in L^{2}(\Omega)$, then the solution of the initial value problem (1.1) and (1.2) is also in $L^{2}(\Omega)$.

Proof. In order to prove this lemma, we divide the proof into two steps.

Firstly, we consider the case of $n=1$, then (1.1) can be rewritten as

$$
{ }_{0} D_{t}^{\alpha_{1}} y(t)=f(t)
$$

Taking the transform of (3.1) to both sides, we obtain

$$
y(t)={ }_{0} D_{t}^{-\alpha_{1}} f(t) .
$$

By virtue of the functions $f(t) \in L^{2}(\Omega)$ and $D^{-\alpha}: L^{2}(\Omega) \rightarrow L^{2}(\Omega)$ that is a bounded linear operator (see [15]), we can easily derive the function $y(t) \in L^{2}(\Omega)$.

And then, let us consider the case of $n>1$.

To (1.1), using the formula (2.2) and taking the Laplace transform to both sides, we conclude that

$$
\left(b_{1} s^{\alpha_{1}}+b_{2} s^{\alpha_{2}}+\cdots+b_{n} s^{\alpha_{n}}\right) Y(s)=F(s),
$$


and then

$$
Y(s)=\frac{F(s)}{b_{1} S^{\alpha_{1}}+b_{2} s^{\alpha_{2}}+\cdots+b_{n} S^{\alpha_{n}}}=F(s) G(s) .
$$

To prove the functions $y(t) \in L^{2}(\Omega)$, we change the functions $G(s)$ into the following form:

$$
G(s)=\frac{1}{b_{n} S^{\alpha_{n}}+b_{n-1} S^{\alpha_{n-1}}} \frac{1}{1+\left(\sum_{k=0}^{n-2} b_{k} S^{\alpha_{k}} /\left(b_{n} S^{\alpha_{n}}+b_{n-1} S^{\alpha_{n-1}}\right)\right)} .
$$

Then replacing the factor $1 /\left(b_{n} s^{\alpha_{n}}+b_{n-1} s^{\alpha_{n-1}}\right)$ with $\left(b_{n}^{-1} s^{-\alpha_{n-1}}\right) /\left(s^{\alpha_{n}-\alpha_{n-1}}+b_{n-1} / b_{n}\right)$ in (3.5), and expanding the second factor into a formal of series, we have

$$
G(s)=\sum_{m=0}^{\infty} \frac{(-1)^{m} b_{n}^{-1} s^{-\alpha_{n-1}}}{\left(s^{\alpha_{n}-\alpha_{n-1}}+b_{n-1} / b_{n}\right)^{m+1}}\left(\sum_{k=0}^{n-2}\left(\frac{b_{k}}{b_{n}}\right) s^{\alpha_{k}-\alpha_{n-1}}\right)^{m} .
$$

Using the method described in [3], we obtain the expression of $G(s)$, which is

$$
G(s)=\frac{1}{b_{n}} \sum_{m=0}^{\infty}(-1)^{m} \sum_{\substack{k_{0}+k_{1}+\cdots+k_{n-2}=m \\ k_{0}, k_{1}, \ldots, k_{n-2} \geq 0}}\left(m ; k_{0}, k_{1}, \ldots, k_{n-2}\right) \prod_{i=0}^{n-2}\left(\frac{b_{i}}{b_{n}}\right)^{k_{i}} \frac{s^{-\alpha_{n-1}+\sum_{i=0}^{n-2}\left(\alpha_{i}-\alpha_{n-1}\right) k_{i}}}{\left(s^{\alpha_{n}-\alpha_{n-1}}+b_{n-1} / b_{n}\right)^{m+1}}
$$

Substituting $G(s)$ in (3.4), considering the formula (2.8), and taking the inverse Laplace transform term-by-term, we obtain that the analytical solution of initial values problem (1.1) and (1.2) in the following form:

$$
y(t)=f(t) * g(t)
$$

with

$$
\begin{aligned}
g(t)= & \frac{1}{b_{n}} \sum_{m=0}^{\infty} \frac{(-1)^{m}}{m !} \sum_{\substack{k_{0}+k_{1}+\cdots+k_{n-2}=m \\
k_{0}, k_{1}, \ldots, k_{n-2} \geq 0}}\left(m ; k_{0}, k_{1}, \ldots, k_{n-2}\right) \prod_{i=0}^{n-2}\left(\frac{b_{i}}{b_{n}}\right)^{k_{i}} t^{\left(\alpha_{n}-\alpha_{n-1}\right) m+\alpha_{n}+\sum_{j=0}^{n-2}\left(\alpha_{n-1}-\alpha_{j}\right) k_{j}-1} \\
& \times E_{\alpha_{n}-\alpha_{n-1}, \alpha_{n}+\sum_{j=0}^{n-2}\left(\alpha_{n-1}-\alpha_{j}\right) k_{j}}^{(m)}\left(-\frac{b_{n-1}}{b_{n}} t^{\alpha_{n}-\alpha_{n-1}}\right)
\end{aligned}
$$

where $\left(m ; k_{0}, k_{1}, \ldots, k_{n-2}\right)=m ! / \prod_{i=0}^{n-2}\left(k_{i} !\right)$ is the multinomial coefficient, the representation of $f(t) * g(t)$ is the convolution of functions $f(t)$ and $g(t)$, and $E_{\lambda, \mu}^{(k)}$ is the $k$ th derivative of the Mittag-Leffler function with parameters $\lambda$ and $\mu$ (see [16]). 
From the representation (3.9), the function $E_{\alpha, \beta}^{(m)}(z)$ is bounded in $z \in C$, and the index satisfies $\left(\alpha_{n}-\alpha_{n-1}\right) m+\alpha_{n}+\sum_{j=0}^{n-2}\left(\alpha_{n-1}-\alpha_{j}\right) k_{j}-1 \geq 0$; so we can easily gain that the function $g(t)$ is bounded in $\Omega$. And then

$$
\|y(t)\|_{L^{2}(\Omega)}=\|f(t) * g(t)\|_{L^{2}(\Omega)} \leq\|f(t)\|_{L^{2}(\Omega)}\|g(t)\|_{L^{2}(\Omega)} \leq C\|f(t)\|_{L^{2}(\Omega)}
$$

it yields that the functions $y(t) \in L^{2}(\Omega)$, and so we have finished the proof of the lemma.

\subsection{The Proof of Existence and Uniqueness}

Considering the solution of the fractional differential equation $y(t) \in L^{2}(\Omega)$ and the fractional B-spline wavelet $\varphi_{+}^{\alpha}(x)$ which generates a Riesz basis for $L^{2}(\Omega)$, we can prove the following theorem.

Theorem 3.2. Let $\alpha_{n}>\alpha_{n-1}>\cdots>\alpha_{1}$, and $\alpha_{n} \geq 1$, and $f(t) \in L^{2}(\Omega)$, then the initial value problems (1.1) and (1.2) have a unique solution. Further more, the solution has explicit representation of fractional B-splines wavelets series.

Proof. From Lemma 3.1, we have obtained the functions $y(t) \in L^{2}(\Omega)$, which can be expressed as

$$
y(t)=\sum_{k=-\infty}^{+\infty} c(k) \varphi_{+}^{\alpha}(t-k)
$$

where the index satisfies $\alpha>0$.

Then substituting (2.14) into (1.1), and taking the Fourier transform to both sides, we obtain

$$
\hat{c}(\omega)\left[b_{1}(i \omega)^{\alpha_{1}}+b_{2}(i \omega)^{\alpha_{2}}+\cdots+b_{n}(i \omega)^{\alpha_{n}}\right] \hat{\varphi}_{+}^{\alpha}(w)=\hat{f}(\omega),
$$

where $\hat{c}(\omega)=\sum_{k=-\infty}^{+\infty} c(k) e^{-i k w}$, and (3.12) is equivalent to the following form:

$$
\hat{c}(\omega) \hat{\psi}_{+}^{\alpha}(w)=\frac{\hat{f}(\omega)}{\left[b_{1}(i \omega)^{\alpha_{1}}+b_{2}(i \omega)^{\alpha_{2}}+\cdots+b_{n}(i \omega)^{\alpha_{n}}\right]} .
$$

And then, by taking the inverse Fourier transform to (3.13), we have

$$
\sum_{k=-\infty}^{+\infty} c(k) \varphi_{+}^{\alpha}(t-k)=f(t) * g(t)
$$

Because the function $\varphi_{+}^{\alpha}(t)$ is the orthogonal fractional B-splines, hence, the representation of the coefficient $c(k)$ is defined by

$$
c(k)=\left\langle f(t) * g(t), \varphi_{+}^{\alpha}(t-k)\right\rangle,
$$


where $\langle\bullet, \bullet\rangle$ is the inner product. Then substituting (3.15) in (2.14), we obtain the solution of the initial value problems (1.1) and (1.2).

Let us suppose that the initial value problems (1.1) and (1.2) have another solution, which can be expressed as

$$
y_{1}(t)=\sum_{n=-\infty}^{+\infty} d(n) \varphi_{+}^{\alpha}(t-n)
$$

Utilizing the similar scheme to $c(k)$, we acquire the representation of $d(n)$, which is

$$
d(n)=\left\langle f(t) * g(t), \varphi_{+}^{\alpha}(t-n)\right\rangle .
$$

Taking (3.17) into (3.16), we obtain the representation of $y_{1}(t)$. Obviously, we have

$$
y(t)-y_{1}(t)=\sum_{k=-\infty}^{+\infty} c(k) \varphi_{+}^{\alpha}(t-k)-\sum_{n=-\infty}^{+\infty} d(n) \varphi_{+}^{\alpha}(t-n)=0
$$

it derives that $y(t)=y_{1}(t)$, which means that the solution of the initial value problems (1.1) and (1.2) is unique. Then substituting $y_{1}(t)$ with its coefficient in (1.1), we can easily check that the equation is correct. It indicates that the function $y_{1}(t)$ is a solution of the differential equation (1.1), which yields the existence of solution. Finally, we have completed the proof of the solution of existence and uniqueness of the nonhomogeneous linear differential equations of arbitrary order.

\section{The Asymptotic Solution of the Fractional Differential Equations and Error Estimation}

\subsection{The Asymptotic Solution of the Fractional Differential Equations}

The purpose of this section is to discuss the case of $\alpha_{i} \in Q^{+}$in (1.1), which appeared in most practical applications we have encountered, and show the asymptotic solution of the fractional differential equations and error estimation. In fact, the case of $\alpha_{i} \in Q, i=1,2, \ldots, n$ in Theorem 3.2 is equivalent to the following corollary.

Corollary 4.1. Let $m v \geq 1$, and $f(t) \in L^{2}(\Omega)$, then the nonhomogeneous linear fractional differential equations

$$
\left(a_{m} D^{m v}+a_{m-1} D^{(m-1) v}+\cdots+a_{0} D^{0}\right) y(t)=f(t)
$$

on the initial values

$$
\left[D^{(m-k) v-1} y(t)\right]_{t=0}=0,\left[D^{(m-k) v-2} y(t)\right]_{t=0}=0, \ldots,\left[D^{(m-k) v-r_{k}-1} y(t)\right]_{t=0}=0,
$$

where $0 \leq r_{k}-1 \leq(m-k) v<r_{k}, v \in Q^{+}, r_{k} \in N, k=0,1, \ldots, m$, have a unique solution. 
In Section 3, we have proved the existence and uniqueness of the nonhomogeneous linear fractional differential equations of arbitrary positive real order. Obviously, it also satisfies the case of positive rational order. To (1.1), let $q \in Z$ be the least common multiple of the denominators of $\alpha_{i} \in Q, i=1,2, \ldots, n$, so $\alpha_{i}=q_{i} / q$, where $q_{i} \in Z, i=1,2, \ldots, n$. And to (4.1), let $v=1 / q$ and $m-i_{0}=q_{n}, m-i_{1}=q_{n-1}, \ldots, m-i_{n-1}=q_{1}$, then considering the conditions of $a_{m-i_{j}}=b_{n-j}, a_{k}=0, k \neq m-i_{j}$, where $i_{j}<m, i_{j} \in Z, j=1,2, \ldots, n-1$. Hence, the initial value problems (4.1) and (4.2) have been changed into of the problems (1.1) and (1.2) with the order $\alpha_{i} \in Q^{+}$, respectively, which means that Theorem 3.2 in the case of $\alpha_{i} \in Q^{+}$ is equivalent to Corollary 4.1 .

Similarity to the scheme of Theorem 3.2, we can easily prove Corollary 4.1 and give the representation of explicit solution of the initial value problems (4.1) and (4.2), which can be defined by

$$
y(t)=\sum_{k=-\infty}^{+\infty} c(k) \varphi_{+}^{\alpha}(t-k)
$$

where $c(k)=\left\langle f(t) * g(t), \varphi_{+}^{\alpha}(t-k)\right\rangle$, and $g(t)$ is the inverse of the Laplace Transform of the function $G(s)$, where

$$
G(s)=\frac{1}{a_{m} s^{m v}+a_{m-1} s^{(m-1) v}+\cdots+a_{0}} .
$$

To obtain the asymptotic solution of (4.1), we will give the explicit formulation of the function $g(t)$. Let $P\left(s^{v}\right)=a_{m} s^{m v}+a_{m-1} s^{(m-1) v}+\cdots+a_{0}$ and $x=s^{v}$; thus the $P(x)$ is a polynomial of the degree $m$. Moreover, we suppose that $\gamma_{1}, \gamma_{2}, \ldots, \gamma_{j}$ are distinct zeros of $P(x)$ with the order of $l_{1}, l_{2}, \ldots, l_{j}$, respectively; by applying the theories of polynomials, the $P(x)$ can be rewritten in the following form

$$
P(x)=a_{m}\left(x-\gamma_{1}\right)^{l_{1}}\left(x-\gamma_{2}\right)^{l_{2}} \cdots\left(x-\gamma_{j}\right)^{l_{j}},
$$

where $\sum_{i=1}^{j} l_{i}=m, j, l_{i} \in N, i=1,2, \ldots, j$, Then, substituting the function $P(x)$ in (4.2) and expanding it to the sum of partial fractions, we have

$$
G(s)=\sum_{i=1}^{j} \sum_{n=0}^{l_{i}-1} \frac{A_{i, l_{i}-n}}{\left(s^{v}-\gamma_{i}\right)^{l_{i}-n}},
$$

where the coefficients $A_{i, l_{i}-n}, i=1,2, \ldots, j ; n=0,1, \ldots, l_{i}-1$, are constants.

Thus, taking the inverse Laplace Transform to (4.6) and using the formulation (2.8), we obtain explicit formulation of the function $g(t)$, defined by

$$
g(t)=\sum_{i=1}^{j} \sum_{n=0}^{l_{i}-1} \frac{t^{(i+1) v-1}}{\left(l_{i}-n\right) ! A_{i, l_{i}-n}} E_{v, v}^{\left(l_{i}-n-1\right)}\left(\gamma_{i} t^{v}\right) .
$$


According to Unser and Blu in [13], the wavelet base generated by fractional B-splines wavelet $\beta_{+}^{\alpha}(x)$ is denseness of the representation in $L^{2}(\Omega)$. Let $\dot{\beta}_{+}^{\alpha}(x)$ be the inverse of Fourier Transform of $\hat{\hat{\beta}}_{+}^{\alpha}(\omega)$, where

$$
\hat{\hat{\beta}}_{+}^{\alpha}(\omega)=\frac{\sqrt{2} \hat{\beta}_{+}^{\alpha}(w)}{\left(\sum_{k=0}^{\infty}\left|\hat{\beta}_{+}^{\alpha}(\omega+2 k \pi)\right|^{2}\right)^{1 / 2}}
$$

With the help of the theories of wavelet analyses, we know that the functions $\dot{\beta}_{+}^{\alpha}(x)$ are an orthogonal fractional B-splines wavelet and dense in $L^{2}(\Omega)$ with $\alpha>0$. Thus the solutions of initial value problems (4.1) and (4.2) have the following form:

$$
y(t)=\sum_{k=-\infty}^{+\infty} c(k) \dot{\beta}_{+}^{\alpha}(t-k)
$$

Then substituting $y(t)$ in (4.1), we obtain the representation of the coefficients $c(k)$, defined by

$$
c(k)=\left\langle f(t) * g(t), \dot{\beta}_{+}^{\alpha}(t-k)\right\rangle .
$$

Finally, by combining (4.9) and (4.10), the obtained function $y(t)$ is the asymptotic solution of initial value problems (4.1) and (4.2) that we are looking for.

\subsection{Order of the Error Estimation}

To estimate the error of asymptotic solution of initial value problems (4.1) and (4.2), we introduce the following properties of the fractional B-spines $\beta_{+}^{\alpha}(x)$, which have been proved by Unser and Blu (see [13, Theorem 3.1]).

Proposition 4.2. For all $\alpha>0$, we have

$$
\beta_{+}^{\alpha}(x)=\frac{\Gamma(\alpha+2) \sin \pi \alpha}{\pi x^{\alpha+2}} \sum_{n \geq 1} \frac{e^{2 n i \pi x}}{(2 n i \pi)^{\alpha+1}}+o\left(\frac{1}{x^{\alpha+2}}\right)
$$

when $x$ tends to $+\infty$.

To (4.8), the function $a(\omega)=\sum_{k=0}^{\infty}\left|\hat{\beta}_{+}^{\alpha}(\omega+2 k \pi)\right|^{2}$ is $2 \pi$-periodic and symmetric, and so we can restrict its study to $\omega \in[0, \pi]$. In particular, one has

$$
a(\omega) \geq\left|\sin c \frac{\omega}{2}\right|^{2 \alpha+2} \geq\left(\frac{2}{\pi}\right)^{2 \alpha+2},
$$

since $\sin c \omega / 2$ is strictly decreasing over $[0, \pi]$ (see [13]). 
International Journal of Differential Equations

Combining (4.12) and (4.8) and taking the inverse of Fourier Transform, we obtain

$$
\dot{\beta}_{+}^{\alpha}(t-k) \leq\left(\frac{2}{\pi}\right)^{\alpha+1} \beta_{+}^{\alpha}(t-k)
$$

Then according to (4.10), (4.11), and (4.13), the asymptotic solution $y(t)$ can be defined by

$$
y(t)=\sum_{k=-\infty}^{+\infty}\left\langle f(t) * g(t), \dot{\beta}_{+}^{\alpha}(t-k)\right\rangle \dot{\beta}_{+}^{\alpha}(t-k) \leq\|f(t) * g(t)\|_{L^{2}} \sum_{k=-\infty}^{+\infty}\left|\dot{\beta}_{+}^{\alpha}(t-k)\right|^{2} .
$$

To calculate the truncated error of the asymptotic solution, let $y_{N}(t)$ be the truncated sum $(|k| \leq N)$ corresponding to asymptotic solution (4.8), where

$$
y_{N}(t)=\sum_{k=-N}^{N}\left\langle f(t) * g(t), \dot{\beta}_{+}^{\alpha}(t-k)\right\rangle \dot{\beta}_{+}^{\alpha}(t-k) .
$$

Thus the truncated error $y^{*}(t)$ will be obtained as follows:

$$
\begin{aligned}
y^{*}(t) & =y(t)-y_{N}(t) \\
& =\sum_{|k|>N}\left\langle f(t) * g(t), \dot{\beta}_{+}^{\alpha}(t-k)\right\rangle \dot{\beta}_{+}^{\alpha}(t-k) \\
& \leq\|f(t) * g(t)\| \sum_{|k|>N}\left|\dot{\beta}_{+}^{\alpha}(t-k)\right|^{2},
\end{aligned}
$$

where the function $g(t)$ is bounded in $\Omega$ and $f(t) \in L^{2}(\Omega)$; with the help of (4.12), the inequality of (4.16) can be amplified, which means that

$$
y^{*}(t) \leq\left(\frac{2}{\pi}\right)^{2 \alpha+2}\|f(t) * g(t)\| \sum_{|k|>N}\left|\beta_{+}^{\alpha}(t-k)\right|^{2}
$$

Then substituting (4.10) in (4.16), we have

$$
y^{*}(t) \leq\left(\frac{2}{\pi}\right)^{2 \alpha+2}\|f(t) * g(t)\| \sum_{|k|>N}\left|\frac{\Gamma(\alpha+2) \sin \pi \alpha}{\pi(t-k)^{\alpha+2}} \sum_{n \geq 1} \frac{e^{2 \pi i n x}}{(2 n i \pi)^{\alpha+1}}+o\left(\frac{1}{(t-k)^{\alpha+2}}\right)\right|^{2} .
$$

To the right side of (4.17), we divided the representation into three parts for discussion. Firstly, because the function $g(t)$ is bounded in $\Omega$ and $f(t) \in L^{2}(\Omega)$, there exists a constant $C_{1}$ which holds

$$
\|f(t) * g(t)\|_{L^{2}} \leq c\|f(t)\|_{L^{2}} \leq C_{1} .
$$


Secondly, we consider the series function

$$
\left|\sum_{n \geq 1} \frac{e^{2 \pi i n x}}{(2 n i \pi)^{\alpha+1}}\right|^{2} \leq \sum_{n \geq 1}\left|\frac{e^{2 \pi i n x}}{(2 n i \pi)^{\alpha+1}}\right|^{2} \leq \sum_{n \geq 1} \frac{1}{(2 n \pi)^{2 \alpha+2}}
$$

which is convergent in $\Omega$; thus there exists a constant $C_{2}$ which satisfies

$$
\left|\sum_{n \geq 1} \frac{e^{2 \pi i n x}}{(2 n i \pi)^{\alpha+1}}\right|^{2} \leq C_{2}
$$

At last, we denote a constant $C_{3}$ which defined by

$$
C_{3}=\frac{2^{2 \alpha+2} \Gamma^{2}(\alpha+2) \sin ^{2} \pi \alpha}{\pi^{2 \alpha+4}} .
$$

Then combining (4.19), (4.21), and (4.22), the inequality of (4.18) can be amplified to the following form:

$$
y^{*}(t) \leq C_{1} C_{2}^{2} C_{3} \sum_{|k|>N}\left|\frac{1}{(t-k)^{\alpha+2}}+o\left(\frac{1}{(t-k)^{\alpha+2}}\right)\right|^{2} \leq C_{1} C_{2}^{2} C_{3} \sum_{|k|>N}\left|\frac{1+C_{0}}{(t-k)^{\alpha+2}}\right|^{2},
$$

when $|t-k|$ tends to $+\infty$.

Thus, from the above discussion, it is evident to derive that the truncated error converges as $\sum_{|k|>N} C /(k-t)^{2 \alpha+4}$, where $C=C_{1} C_{2}^{2} C_{3}\left(1+C_{0}\right)^{2}$. Note that we should choose a suitable $N$ which should be much more greater than $T$, where, $t \in \Omega=[0, T]$. Finally, we have obtained the error order of the asymptotic truncated sum in theory.

Remark 4.3. Noting that the process of the proof of the existence and uniqueness of the solution of the initial value problems (1.1) and (1.2) in the case of Riemann-Liouville fractional differential operator, and considering the formula of the Laplace transform of Caputo differential operator, we can be easily replaced the case of Riemann-Liouville fractional differential operator by Caputo sense with its initial values. It means the following corollary is correct.

Corollary 4.4. Let $\alpha_{n}>\alpha_{n-1}>\cdots>\alpha_{1}>0$, and $\alpha_{n} \geq 1$, and $f(t) \in L^{2}(\Omega)$, then the multiterms fractional ordinary differential equations

$$
\left(b_{n} D^{\alpha_{n}}+b_{n-1} D^{\alpha_{n-1}}+\cdots+b_{1} D^{\alpha_{1}}\right) y(t)=f(t)
$$

with its initial values

$$
y^{(k)}(0)=0, \quad\left(k=0,1,2, \ldots,\left\lceil\alpha_{n}\right\rceil-1\right)
$$

where $\left[\alpha_{n}\right]=\max \left\{m \mid m \leq \alpha_{n}, m \in Z\right\}$, have a unique solution. 


\section{Conclusion}

In this paper, we have proved the existence and uniqueness of the solution of the differential equations of arbitrary positive real order. And the representation of the solution of (1.1) has been given in the process of proof. We have obtained the asymptotic solution of the differential equations of fractional order $\alpha \in Q^{+}$and corresponding error estimation. The most notable feature is the order of the asymptotic truncated error, namely, $(2 \alpha+4)$ th, which is effective to calculate the numerical solution of (4.1). In particular, the case of Riemann-Liouville differential operator is replaced by Caputo sense with its initial values in Corollary 4.4. Similarly to the scheme of the proof as showen in the paper, by considering the relationship between Riemann-Liouville and Caputo differential operator, we can easily complete the proof of the corollary in the case of the Caputo differential operator, which shows that the method we have discussed can be applied more widely.

\section{References}

[1] P. Kumar and O. P. Agrawal, "Numerical scheme for the solution of fractional differential equations of order greater than one," Journal of Computational and Nonlinear Dynamics, vol. 1, no. 2, 8 pages, 2006.

[2] K. S. Miller and B. Ross, An Introduction to the Fractional Calculus and Fractional Differential Equations, A Wiley-Interscience Publication, John Wiley \& Sons, New York, NY, USA, 1993.

[3] I. Podlubny, Fractional Differential Equations, vol. 198 of Mathematics in Science and Engineering, Academic Press, San Diego, Calif, USA, 1999.

[4] J. T. Edwards, N. J. Ford, and A. C. Simpson, "The numerical solution of linear multi-term fractional differential equations; systems of equations," Journal of Computational and Applied Mathematics, vol. 148, no. 2, pp. 401-418, 2002.

[5] K. Diethelm and N. J. Ford, "Analysis of fractional differential equations," Journal of Mathematical Analysis and Applications, vol. 265, no. 2, pp. 229-248, 2002.

[6] K. Diethelm, N. J. Ford, and A. D. Freed, "A predictor-corrector approach for the numerical solution of fractional differential equations," Nonlinear Dynamics, vol. 29, no. 1-4, pp. 3-22, 2002.

[7] K. Diethelm, "Efficient solution of multi-term fractional differential equations using $P(E C)^{\mathrm{m}} E$ methods," Computing, vol. 71, no. 4, pp. 305-319, 2003.

[8] C. Yang and F. Liu, "A computationally effective predictor-corrector method for simulating fractional order dynamical control system," The ANZIAM Journal, vol. 47, pp. C168-C184, 2005.

[9] K. Diethelm and N. J. Ford, "Multi-order fractional differential equations and their numerical solution," Applied Mathematics and Computation, vol. 154, no. 3, pp. 621-640, 2004.

[10] V. Daftardar-Gejji and A. Babakhani, "Analysis of a system of fractional differential equations," Journal of Mathematical Analysis and Applications, vol. 293, no. 2, pp. 511-522, 2004.

[11] M. Unser and T. Blu, "Construction of fractional spline wavelet bases," in Wavelets Applications in Signal and Image Processing VII, vol. 3813 of Proceedings of SPIE, pp. 422-431, Denver, Colo, USA, July 1999.

[12] S. Mallat, A Wavelet Tour of Signal Processing, Academic Press, San Diego, Calif, USA, 2nd edition, 1998.

[13] M. Unser and T. Blu, "Fractional splines and wavelets," SIAM Review, vol. 42, no. 1, pp. 43-67, 2000.

[14] M. Unser, A. Aldroubi, and M. Eden, "A family of polynomial spline wavelet transforms," Signal Processing, vol. 30, pp. 141-162, 1993.

[15] V. J. Ervin and J. P. Roop, "Variational formulation for the stationary fractional advection dispersion equation," Numerical Methods for Partial Differential Equations, vol. 22, no. 3, pp. 558-576, 2005.

[16] F. Mainardi and R. Gorenflo, "On Mittag-Leffler-type functions in fractional evolution processes," Journal of Computational and Applied Mathematics, vol. 118, no. 1-2, pp. 283-299, 2000. 


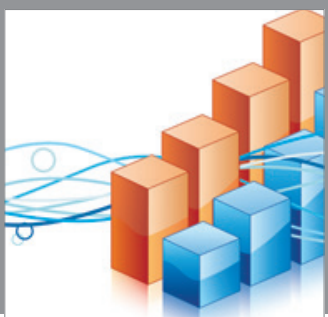

Advances in

Operations Research

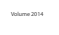

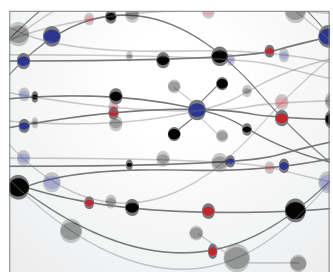

\section{The Scientific} World Journal
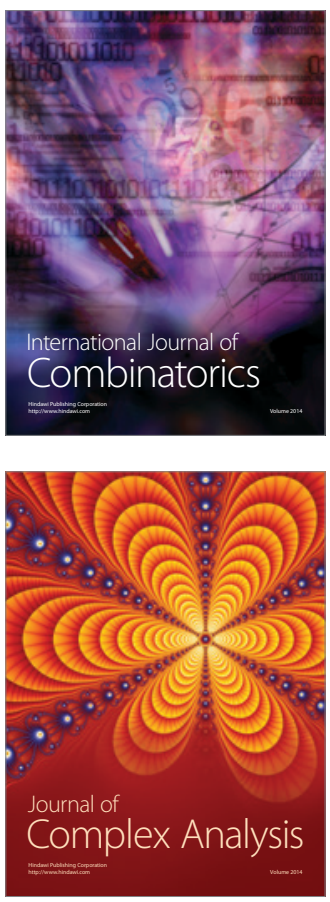

International Journal of

Mathematics and

Mathematical

Sciences
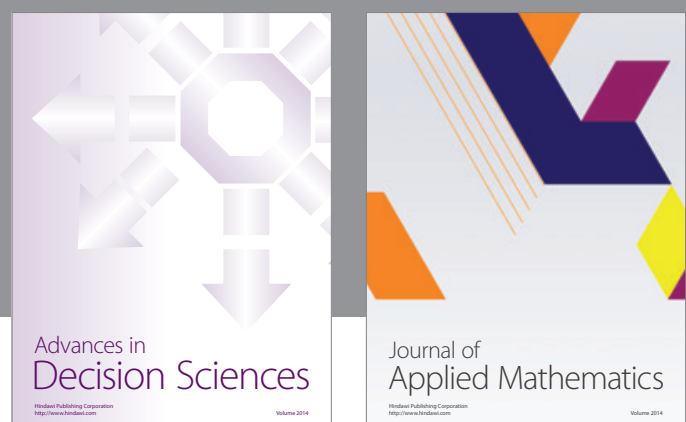

Journal of

Applied Mathematics
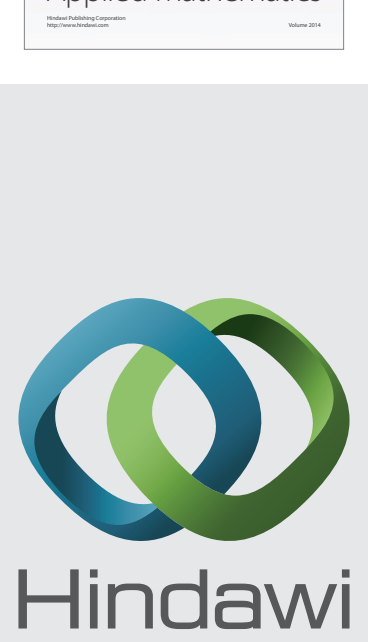

Submit your manuscripts at http://www.hindawi.com
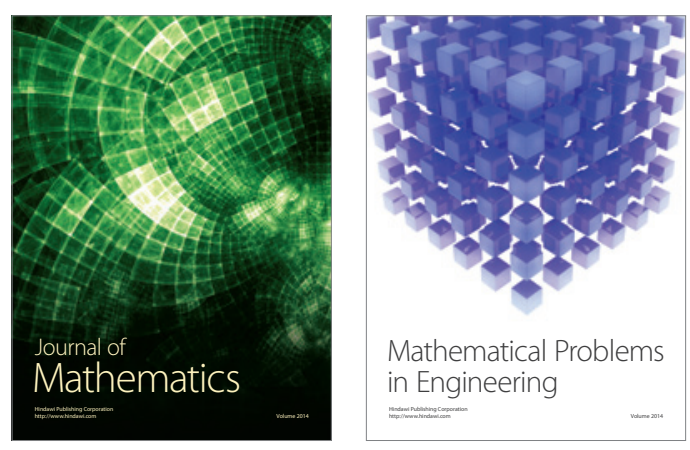

Mathematical Problems in Engineering
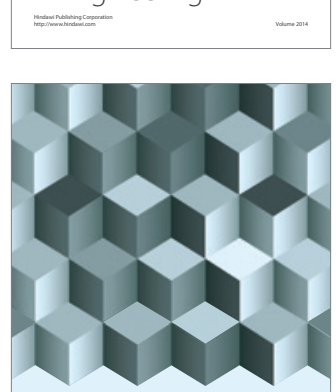

Journal of

Function Spaces
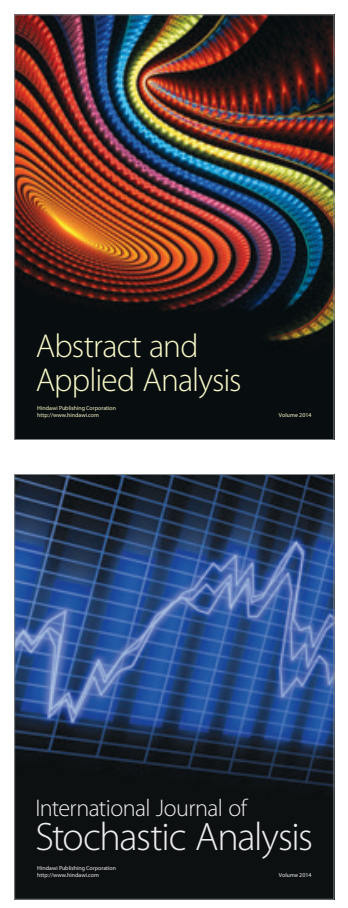

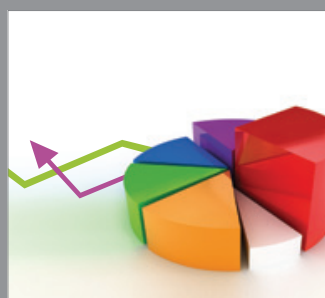

ournal of

Probability and Statistics

Promensencen
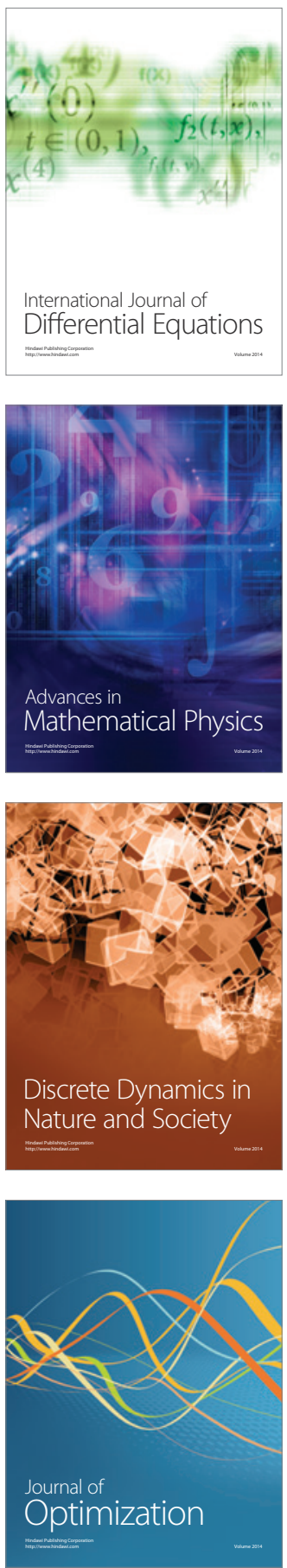